\title{
Erratum to: Influence of Braid Carrier Tension on Carbon Fibre Braided Preforms
}

\author{
Sree Shankhachur Roy, Wentao Zou and Prasad Potluri
}

\section{Erratum to:}

Chapter 'Influence of Braid Carrier Tension on Carbon Fibre Braided Preforms' in: Y. Kyosev (ed.), Recent Developments in Braiding and Narrow Weaving, DOI 10.1007/978-3-319-29932-7_9

The book was inadvertently published with an incorrect surname of the author as "Wentao Zao" in Chapter "Influence of Braid Carrier Tension on Carbon Fibre Braided Preforms' whereas the correct surname is "Wentao Zou". The erratum chapter and the book has been updated for the same.

The updated original online version for this chapter can be found at DOI 10.1007/978-3-319-29932-7_9

\footnotetext{
S.S. Roy $(\bowtie) \cdot$ W. Zou $\cdot$ P. Potluri

Textile Composites Group, The University of Manchester, Manchester, UK

e-mail: shankhachur.roy@manchester.ac.uk 